\title{
Can rotated words be processed automatically? Evidence from rotated repetition priming
}

\author{
András Benyhe $^{1}$ (i) $\cdot$ Péter $^{C}$ Ssibri $^{1}$ \\ Accepted: 28 January 2021 / Published online: 15 March 2021 \\ (C) The Psychonomic Society, Inc. 2021
}

\begin{abstract}
Visual word processing has its own dedicated neural system that, due to the novelty of this activity, is unlikely to have acquired its specialization through natural selection. Understanding the properties of this system could shed light on its recruitment and the background of its disorders. Although recognition of simple visual objects is orientation invariant, this is not necessarily the case for written words. We used a masked repetition priming paradigm to find out whether words retain their readability when viewed in atypical orientations. Subjects had to read out upright target words that were preceded by rotated prime words of the same or different identity. Priming duration was varied in Experiment 1 to assess the temporal emergence of a rotated priming effect. In Experiment 2, the letter order of the prime words was reversed in order to differentiate the processing stage where priming occurs. The orientational pattern of the priming effects seen in our results mostly confirms earlier word recognition models, but also serves a more detailed view about the effects of orientation on word form processing.
\end{abstract}

Keywords Reading $\cdot$ Visual word recognition $\cdot$ Rotation $\cdot$ Masked priming

Reading is a relatively recent linguistic activity, invented and implemented by humans in the last few millennia. Its neural bases have been studied since at least the late 19th century (Dejerine, 1892), and a key area was traced to the left occipitotemporal cortex, a region later termed the visual word form area (VWFA; Cohen et al., 2000; Dehaene \& Cohen, 2011; Martin, 2006; Warrington \& Shallice, 1980). It is puzzling how a localized cortical area that evolved with humans can serve such a novel, specialized function such as reading. To solve this problem, Dehaene proposed a hypothesis called neuronal recycling, suggesting that new functions could arise in cortical areas that originally serve another purpose, but match the requirements of the new one (Dehaene \& Cohen, 2007). The hypothesized reason for the occipitotemporal localization of the VWFA is twofold: firstly, here, the visual input reaches the level of processing required for orthographic decoding, and secondly, this area has sufficient connectivity with frontotemporal language networks to serve its function (Bouhali et al., 2014; Kim, Kanjlia, Merabet, \& Bedny, 2017; Reich, Szwed, Cohen, \& Amedi, 2011).

András Benyhe

benyheandras@gmail.com

1 Department of Physiology, University of Szeged Faculty of Medicine, Szeged, Hungary
One of the most accepted frameworks of visual word recognition is the local combinations detector (LCD) model. This states that the hierarchical organization of the ventral visual stream can be applied to the processing of written words: line segments are combined into small local patterns, those into letters, groups of letters, and then finally, words (Dehaene, Cohen, Sigman, \& Vinckier, 2005). Invariance to text size, position, and other aesthetic parameters like case and font can arise along this stream, as receptive field size and preferred stimulus complexity grows. Subsequent areas of the stream deal with increasingly complex information in a parallel manner (i.e., letters of a word are recognized at a single glance and then are fed forward in a combinatorial system, the VWFA, that outputs the most likely word that the letters code; Grainger, 2018; Norris, 2013). This ventral parallel processing allows well-trained readers to read quickly with ease. Evidence suggests, however, that when words are observed out of their usual appearance, the quick parallel processing and decoding are lost and auxiliary mechanisms through the dorsal pathway must salvage recognition in a serial, letter-byletter manner (Cohen, Dehaene, Vinckier, Jobert, \& Montavont, 2008). In-plane rotation is considered such a transformation, and even though letter recognition is not affected by rotation (Koriat \& Norman, 1989; Perea, VergaraMartínez, Marcet, Mallouh, \& Fernández-López, 2020; but see Risko, Medimorec, Chisholm, \& Kingstone, 2014), word recognition is heavily impaired by it (Cohen et al., 2008). The 
common explanation is that rotation breaks the experiencebased functions of the VWFA, and it takes an increased cognitive load to mentally normalize the visual image back to its normal orientation. Yet we might argue that perception of orientation must come afterward shape is recognized, since without knowing what the shape is, we cannot possibly tell how it should be oriented (Corballis \& Armstrong, 2007), and cases of isolated orientation agnosia also hint at this conclusion (Harris, Harris, \& Caine, 2001). Indeed, evidence has been found of an early, orientation-invariant priming effect in object recognition (Harris, Dux, Benito, \& Leek, 2008), but its validity in reading has not yet been studied in great detail; although some specific studies in recent years pointed out that priming is possible using marquee and $90^{\circ}$ rotated words (Perea, Marcet, \& Fernández-López, 2018; Witzel, Qiao, \& Forster, 2011) and rotated letters (Perea et al., 2020).

To understand the detailed neurophysiological background of reading, it is essential to find the limitations and requirements of the system that carries it out. The goal of this study was to find out whether orientation-invariant priming exists for word recognition and also to investigate which aspects of written words are necessary to elicit such an effect.

\section{Experiment 1: Rotated word priming}

To gather detailed data on rotated word processing, we set out to implement the rotated repetition priming design of Harris et al. (2008), using words instead of images. In this paradigm, primes are rotated and targets are presented upright, highlighting the automaticity of orientation invariance in recognition. We chose to keep the naming task from that work, as it seemed more direct and thus more sensitive than a lexical decision.

\section{Materials and methods}

\section{Procedure}

Participants were seated $57 \mathrm{~cm}$ from the viewing screen, with a microphone in front of them. Their task was to read target words presented to them out loud, as quickly as possible. A total of 224 trials were presented in a single run, lasting approximately 10 minutes. Each trial started with a central fixation cross presented for an irregular interval between 1,500 and 2,000 ms. This was followed by a lowercase prime stimulus that varied in its identity and orientation and was of a different duration for subject groups (discussed in the Design section). Following the prime, a mask, composed of scattered characters, was presented for $100 \mathrm{~ms}$. Finally, the uppercase target stimulus was displayed until response. The computer recorded voice onset and paced the experiment automatically. Reaction times (RT) and reading accuracy were later validated off-line by the experimenter, based on voice recordings. Trials with inaccurate utterances, late microphone triggering, and thus incomplete recording or RT above $1,000 \mathrm{~ms}$ were excluded from the analysis. Trials with premature triggering were fixed by resetting the measured RT to the actual start of the response.

\section{Design}

The experiment design (Fig. 1) was based on the first experiment by Harris et al. (2008). The within-subject factors were prime identity and prime orientation. Primes could either be of the same identity as the target they preceded ("same" condition: vonat $\rightarrow$ VONAT) or could be an unrelated word ("different" condition: kalap $\rightarrow$ VONAT). Primes could be presented in any orientation, ranging from $0^{\circ}$ to $360^{\circ}$ in $30^{\circ}$ steps. The duration of the prime stimulus was a between-subject

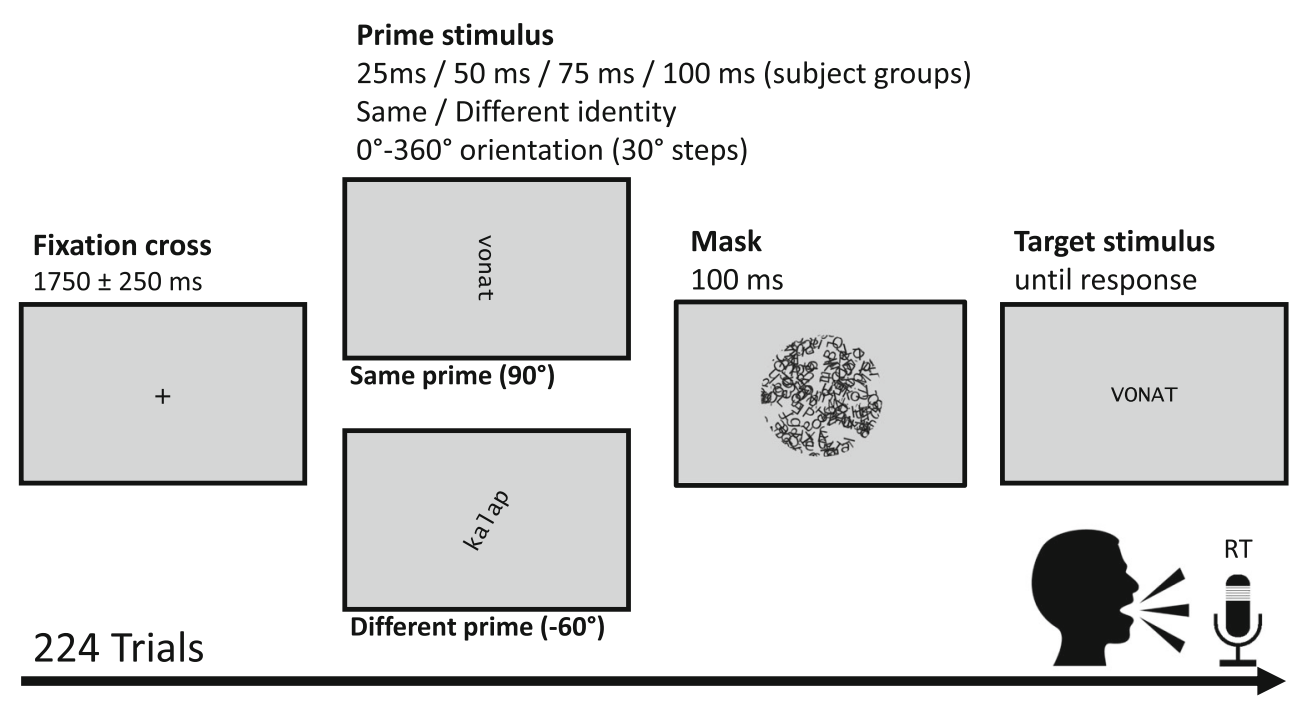

Fig. 1 The design of Experiment 1 (detailed in text) 
factor and was one of the following: $25,50,75,100$. The prime-target stimulus-onset asynchrony (SOA) was therefore $125,150,175$, and $200 \mathrm{~ms}$ for these groups, with the priming duration added to the fixed-length backward mask.

Word order and prime-target pairings were randomized according to Harris et al. (2008), with each pair being unique and each target only occurring once per experiment. For each prime, an orientation $\left(0^{\circ}-180^{\circ}\right)$ and a direction $( \pm)$ were chosen in a pseudorandom manner. This way, in every orientation, 8 "same" and 8 "different" trials occurred (except for $0^{\circ}$ and $180^{\circ}$ orientations, given the \pm equality, these occurred 16 16 times).

\section{Stimuli}

The stimuli were the 336 most frequent (17-196/million) Hungarian nouns, five to six characters in length (Oravecz, Váradi, \& Sass, 2014). Words were drawn on light gray (\#C8C8C8) background using Lucida Console, a monospaced typeface, in black color, and 40-pt font size. Words made up for a $\sim 3.5 \times 0.7^{\circ}$ visual angle. To minimize visual similarity while retaining the orthographic code, primes were presented in lowercase, whereas targets were capitalized.

Masks were designed to disguise both the prime word and its orientation, and hence we had to use a circular mask, composed of visually similar elements as the primes. These were generated at the start of each run by scattering 300 Hungarian characters uniformly on a $400 \times 400$-pixel area and cropping the texture to a circle ( $\sim 7^{\circ}$ visual angle). Five masks were generated and drawn randomly for each trial at random rotation angles to minimize familiarization to masking.

\section{Equipment}

The experiment was carried out in the psychophysics laboratory of our department, in a dark and quiet environment. The experiment was programmed and executed in MATLAB R2016a with Psychophysics Toolbox Version 3.0.14 running under Microsoft Windows (Kleiner, Brainard, \& Pelli, 2007). Stimuli were presented on an Asus PG248Q monitor with $120 \mathrm{~Hz}$ refresh rate and responses were captured with a Rode NT-USB microphone.

\section{Subjects}

Fifty-three healthy young adults ( 34 females, $25.6 \pm 4.6$ years) participated in the study all of whom had normal or corrected to normal vision, reported no reading difficulties, and were naive to the aims and means of the experiment. All participants gave written consent. The procedures were all approved by the Regional Research Ethics Committee of the University of Szeged and were applied in concordance with the World Medical Association Helsinki Declaration.
Group sizes: In a pilot study, we found a relatively large priming effect at normal orientation $(\sim 70 \mathrm{~ms})$ that decayed to zero with increasing orientation. To calculate appropriate sample sizes, we simulated data sets with variable effect sizes at different orientations and variances based on the pilot model and performed the same analyses that we planned to do on the real data. These calculations showed that to achieve $80 \%$ power for priming effects above $20 \mathrm{~ms}$, at any point of the design, we would need 20 participants per group. The COVID-19 outbreak and the consequent regulations, however, prevented us from reaching this goal. Based on the same simulations, we concluded that even with 10 subjects, we would still have the $80 \%$ power margin at around a $30-\mathrm{ms}$ priming effect size, and with 15 subjects at $25 \mathrm{~ms}$. As the systematic steps in priming duration and orientation are fitted into separate effects, any smoothness in the pattern of these would further increase the reliability of the results. We concluded that the available subject numbers $(10,17,16$, and 10 in the four groups, respectively) yield sufficient power in this paradigm.

\section{Analysis}

Analyses were performed in R (Version 3.6.2) using the lme4, and emmeans packages. Separate generalized linear mixedeffects models (GLMM) with inverse Gaussian function and log link were fitted to the RT data from each experimental group (Bates, Mächler, Bolker, \& Walker, 2015; Lenth, Singmann, Love, Buerkner, \& Herve, 2018). Fixed effects were evaluated with Wald tests, and random-effect significance was analyzed with likelihood ratio tests of reduced models. The priming effect for all conditions was calculated as estimated marginal mean contrasts between "same" and "different" conditions. Plots were made with the ggplot package (Wickham, 2016).

\section{Results}

A total of 11,351 RT observations were included in the analysis, and 521 ( $\sim 4 \%$ of total) were excluded on the grounds stated earlier. In the four priming groups $10,17,16$, and 10 subjects were included, respectively.

Separate GLMMs per group were built with prime identity and orientation as discrete fixed-effects with interactions. The natural logarithm of trial number was added as an independent continuous fixed effect. Random intercepts were fitted by subject and target word, and a random effect of prime identity was fitted by subject. All models converged with the bobyqa method included in the lme4 package.

Wald test results of fixed effects significance are summarized in Table 1. Likelihood ratio tests of diminished models justified the random effects structure across the models (see Table 2). 
Table 1 Wald test results on the importance of the denoted fixed effects in the models of Experiments 1 and 2

\begin{tabular}{|c|c|c|c|c|c|c|c|c|c|c|c|}
\hline \multirow[t]{3}{*}{ Fixed effects } & \multirow[t]{3}{*}{$d f$} & \multicolumn{8}{|c|}{ Experiment 1} & \multirow{2}{*}{\multicolumn{2}{|c|}{$\frac{\text { Experiment } 2}{\text { reversed }}$}} \\
\hline & & \multicolumn{2}{|l|}{$25 \mathrm{~ms}$} & \multicolumn{2}{|l|}{$50 \mathrm{~ms}$} & \multicolumn{2}{|l|}{$75 \mathrm{~ms}$} & \multicolumn{2}{|c|}{$100 \mathrm{~ms}$} & & \\
\hline & & $x^{2}$ & $p$ & $x^{2}$ & $p$ & $x^{2}$ & $p$ & $x^{2}$ & $p$ & $x^{2}$ & $p$ \\
\hline prime identity & 1 & 0.59 & .44 & 16.56 & $<.001$ & 42.86 & $<.001$ & 18.77 & $<.001$ & 0.82 & .36 \\
\hline prime orientation & 11 & 13.59 & .26 & 40.95 & $<.001$ & 48.84 & $<.001$ & 10.98 & .45 & 20.83 & 0.035 \\
\hline $\log$ (trial number) & 1 & 2.13 & .14 & 51.32 & $<.001$ & 176.38 & $<.001$ & 83.80 & $<.001$ & 88.34 & $<.001$ \\
\hline \multirow[t]{2}{*}{ prime identity:orientation } & 11 & 44.1 & $<.001$ & 260.28 & $<.001$ & 164.60 & $<.001$ & 77.15 & $<.001$ & 10.52 & .48 \\
\hline & & \multicolumn{2}{|c|}{$n=10(2,191)$} & \multicolumn{2}{|c|}{$n=17(3,651)$} & \multicolumn{2}{|c|}{$n=16(3,379)$} & \multicolumn{2}{|c|}{$n=10(2,130)$} & \multicolumn{2}{|c|}{$n=14(3,037)$} \\
\hline
\end{tabular}

Note. $n$ is the number of subjects with the total number of trials in parentheses.

Marginal means were calculated for "same" and "different" priming conditions in each orientation and priming duration (see Table 3). Priming effect was calculated as the difference between conditions and multiple comparisons were adjusted with the Tukey method. The orientational pattern of priming effect was different across priming durations (see Fig. 2). With the shortest priming duration, it is merely present at $0^{\circ}$ and $-30^{\circ}$, and its size only reaches a few tens of milliseconds. This extends with longer priming durations, and we see a stable and comparable priming effect from $-60^{\circ}$ to $60^{\circ}$ of a size around or above $50 \mathrm{~ms}$. The effect peaks at normal orientation. Curiously, a counterclockwise bias stabilizes across all groups with effects being stronger for rotations in that direction (possibly related to resting head position (Risko et al., 2014), as this was not controlled in our experiments). Also, a priming tendency can be seen at $-150^{\circ}$ with the $50 \mathrm{~ms}$ primes that grows into a significant priming effect at $75-\mathrm{ms}$ duration. Another incidental feature is that with the longest duration there is a relative reduction in the priming effect size around $0^{\circ}$, and much larger variance (even when compared with the 25 -ms group that has the same number of subjects).

\section{Experiment 2: Reversed letter order}

After Experiment 1, we wanted to confirm, that the rotated priming effect is caused by orthographic similarity and not by overlap at lower levels of processing. For this reason, we decided to repeat the experiment using reversed primes with 50-ms duration, as this was the shortest, where the wider orientation-specific pattern arose.

\section{Materials and methods}

\section{Procedure and design}

Experiment 2 was an exact duplicate of the $50 \mathrm{~ms}$ prime duration group of Experiment 1, with one modification: the letter order of prime words was reversed (e.g., tanov $\rightarrow$ VONAT).

\section{Subjects}

Fourteen people (nine females, $21.7 \pm 2.7$ years) matching the same criteria as in Experiment 1 participated under the same ethical license.

\section{Results}

The model was built from 3,037 RT data points from 14 subjects, 99 trials ( $\sim 3 \%$ of total) were rejected. The model structure and fitting methods were identical to the one used in Experiment 1. Wald test and likelihood ratio test results are summarized in Tables 1 and 2.

Table 2 Likelihood ratio test results of diminished models lacking the denoted random effect, compared with the full models of Experiments 1 and 2

\begin{tabular}{|c|c|c|c|c|c|c|c|c|c|c|c|}
\hline \multirow[t]{3}{*}{ Random effects } & \multirow[t]{3}{*}{$d f$} & \multicolumn{8}{|c|}{ Experiment 1} & \multirow{2}{*}{\multicolumn{2}{|c|}{$\begin{array}{l}\text { Experiment } 2 \\
\text { reversed }\end{array}$}} \\
\hline & & \multicolumn{2}{|l|}{$25 \mathrm{~ms}$} & \multicolumn{2}{|l|}{$50 \mathrm{~ms}$} & \multicolumn{2}{|l|}{$75 \mathrm{~ms}$} & \multicolumn{2}{|l|}{$100 \mathrm{~ms}$} & & \\
\hline & & $\chi^{2}$ & $p$ & $x^{2}$ & $p$ & $\chi^{2}$ & $p$ & $\chi^{2}$ & $p$ & $\chi^{2}$ & $p$ \\
\hline by subject prime identity & 2 & 2.00 & .37 & 97.25 & $<.001$ & 33.54 & $<.001$ & 47.05 & $<.001$ & 11.30 & .004 \\
\hline \multirow[t]{2}{*}{ by target intercept } & 1 & 146.92 & $<.001$ & 268.35 & $<.001$ & 246.40 & $<.001$ & 126.13 & $<.001$ & 353.67 & $<.001$ \\
\hline & & \multicolumn{2}{|c|}{$n=10(2,191)$} & \multicolumn{2}{|c|}{$n=17(3,651)$} & \multicolumn{2}{|c|}{$n=16(3,379)$} & \multicolumn{2}{|c|}{$n=10(2,130)$} & \multicolumn{2}{|c|}{$n=14(3,037)$} \\
\hline
\end{tabular}

Note. $n$ is the number of subjects with the total number of trials in parentheses. 
Table 3 RT marginal means and their differences in ms, across prime orientations and conditions in Experiments 1 and 2

\begin{tabular}{|c|c|c|c|c|c|c|c|c|c|c|c|c|c|c|}
\hline & & & \multicolumn{12}{|c|}{ Prime orientation } \\
\hline & & & $0^{\circ}$ & $30^{\circ}$ & $60^{\circ}$ & $90^{\circ}$ & $120^{\circ}$ & $150^{\circ}$ & $180^{\circ}$ & $-150^{\circ}$ & $-120^{\circ}$ & $-90^{\circ}$ & $-60^{\circ}$ & $-30^{\circ}$ \\
\hline \multirow[t]{12}{*}{ Experiment 1} & $25 \mathrm{~ms}$ & same & 470 & 495 & 486 & 484 & 493 & 487 & 497 & 493 & 497 & 503 & 486 & 473 \\
\hline & & different & 500 & 491 & 492 & 486 & 487 & 492 & 486 & 489 & 484 & 491 & 482 & 493 \\
\hline & & priming & $29 * * *$ & -4 & 7 & 2 & -6 & 5 & -11 & -4 & -12 & -12 & -3 & $20^{*}$ \\
\hline & $50 \mathrm{~ms}$ & same & 470 & 480 & 486 & 507 & 515 & 527 & 521 & 514 & 517 & 510 & 484 & 469 \\
\hline & & different & 555 & 549 & 538 & 515 & 518 & 520 & 522 & 532 & 519 & 537 & 537 & 546 \\
\hline & & priming & $85 * * *$ & $69 * * *$ & $51 * * *$ & 8 & 4 & -7 & 1 & 18 & 1 & $27^{* *}$ & $53 * * *$ & $76^{* * * *}$ \\
\hline & $75 \mathrm{~ms}$ & same & 457 & 481 & 488 & 501 & 504 & 505 & 502 & 495 & 503 & 489 & 466 & 458 \\
\hline & & different & 540 & 537 & 528 & 521 & 518 & 516 & 519 & 525 & 514 & 510 & 524 & 538 \\
\hline & & priming & $83 * * *$ & $56 * * *$ & $39 * * *$ & $20 *$ & 13 & 11 & $17^{*}$ & $30 * *$ & 10 & $21 *$ & $58 * * *$ & $80 * * *$ \\
\hline & $100 \mathrm{~ms}$ & same & 471 & 474 & 465 & 497 & 503 & 504 & 494 & 500 & 498 & 483 & 473 & 475 \\
\hline & & different & 534 & 538 & 531 & 520 & 519 & 510 & 520 & 515 & 514 & 512 & 548 & 536 \\
\hline & & priming & $63 * * *$ & $64 * * *$ & $66^{* * * *}$ & $23^{*}$ & 16 & 6 & $25^{*}$ & 15 & 15 & $29^{*}$ & $74 * * *$ & $60 * * *$ \\
\hline \multirow[t]{3}{*}{ Experiment 2} & reversed & same & 512 & 495 & 499 & 494 & 493 & 493 & 492 & 495 & 493 & 488 & 497 & 500 \\
\hline & & different & 495 & 503 & 493 & 488 & 491 & 489 & 492 & 496 & 493 & 487 & 494 & 493 \\
\hline & & priming & $-17 * *$ & 8 & -6 & -5 & -2 & -4 & 0 & 1 & 0 & -1 & -2 & -7 \\
\hline
\end{tabular}

$* p<.05 . * * p<.01 . * * * p<.001$.

Tukey corrected marginal means were contrasted similarly to Experiment 1 (Table 3) and priming effects are plotted in Fig. 3. Only a small inverse priming effect was detected at $0^{\circ}$; otherwise, there was no difference between priming conditions with the reversed prime words.

\section{Discussion}

In the paper that our study was mostly based on, Harris et al. (2008) found that in object recognition, the size of the priming effect increased with priming duration, most apparent above $70 \mathrm{~ms}$ and that it was independent of prime orientation. This orientation invariance is in sharp contrast with our findings, but may be explained by the exceptional role of letters in human visual recognition (Grainger, 2018) and the "unlearning" of mirror-generalization during learning to read (Dehaene et al., 2005).

Repetition priming is believed to occur because the prime, at least partly, preactivates the processing network (Dehaene et al., 2001; Holcomb \& Grainger, 2007). In the case of our paradigm, this network includes the phonological and articulatory systems besides the orthographic and visual ones, as the responses were vocal (Fig. 4). In trials of effective priming, the prime had elicited some activation along the pathway that later processed the target and gave an output. This means that in the conditions where a priming effect was detected, the processing of the prime words was successful, even if they were rotated. The results of Cohen et al. (2008) show that in an unprimed paradigm, lexical decision was markedly slowed by rotation of $90^{\circ}$ (>200 ms for words of length of four to six characters), implying that the recruitment of the dorsal pathway for alternative reading strategies is rather time-consuming. In our experimental design, however, alternative reading strategies are unlikely to be implemented during the short prime-target SOA, and the same applies to conscious mental rotation or perspective-taking as orientation normalization strategies. The randomness of the consequent rotations also excludes the possibility of anticipation-driven orientation normalization, and the small main effect of orientation in the Wald tests also suggests the lack of time-dependent mechanisms. Note that in our experience, the subjects only occasionally reported seeing the primes, mostly in the 100-ms group, solely in the case of "different" primes, and never spotted that orientations other than normal were involved (unregistered data). Based on these findings we conclude, that the priming effect seen in Experiment 1 arises through the highly automated VWFA.

Importantly, the earlier attempts at examining a priming effect with vertically oriented words, employed block designs, with all primes and targets being in the same orientation within a block (Perea et al., 2018; Witzel et al., 2011). This way the subjects are prepared for the orientation of the target, and this anticipation could already affect the processing of the prime since they appear in the same manner. Furthermore, in this setting, individual letters of the prime and target words could spatially overlap in the "same" condition, thus permitting a priming effect at the level of letter detectors. With our method, this only stands for the normal orientation, as the targets were always presented horizontally, but higher orthographic processing is necessary for rotated primes to have an effect. 


\section{5 ms priming}

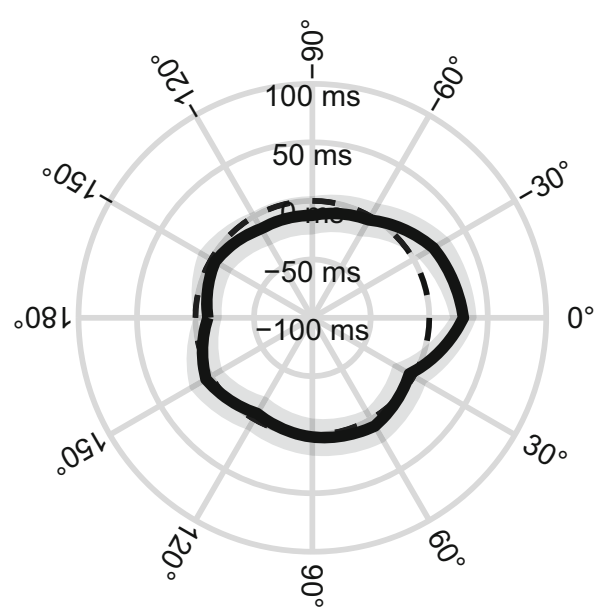

75 ms priming

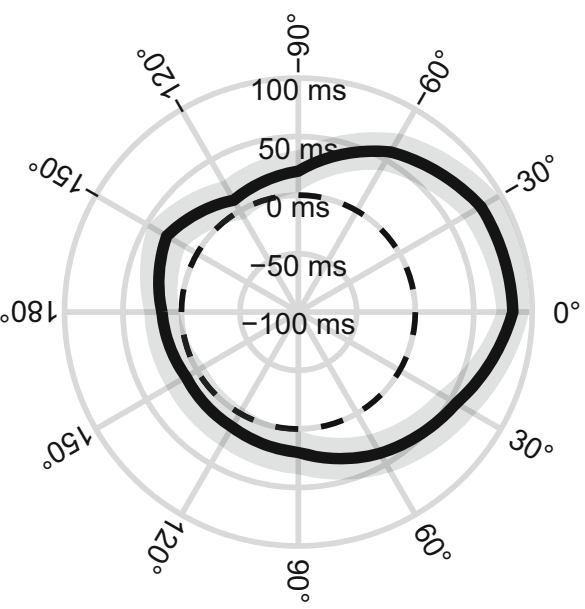

Fig. 2 Priming effect as a function of prime orientation in Experiment 1. RT difference between marginal means of "same" and "different" conditions in $\mathrm{ms} \pm 95 \%$ confidence intervals. The dashed circle is the zero line, meaning no effect, and positive priming effects are observed

We regard that the pattern of rotated priming in Experiment 1 does not conflict with most predictions of the LCD model, but some details call for amendments. Cohen et al. (2008) suggest that the involvement of the VWFA in word form processing has a binary pattern, it is either on or off, and its function breaks after rotation reaches a certain limit $\left(\sim 45^{\circ}\right)$. The narrow effect at 25 -ms priming duration could be attributed to priming at the level of case-invariant letter detectors, as there was a spatial overlap between the letters of the prime and target in the "same" condition. However, as duration increased, so did the priming effect and an orientation invariance emerged between $\pm 60^{\circ}$ rotations with a diminished effect up to $\pm 90^{\circ}$. These results point at a rapid word-level abstraction of rotated primes, attributed to WVFA, meaning that the orientation-invariant boundary could be less sharp and at a
50 ms priming

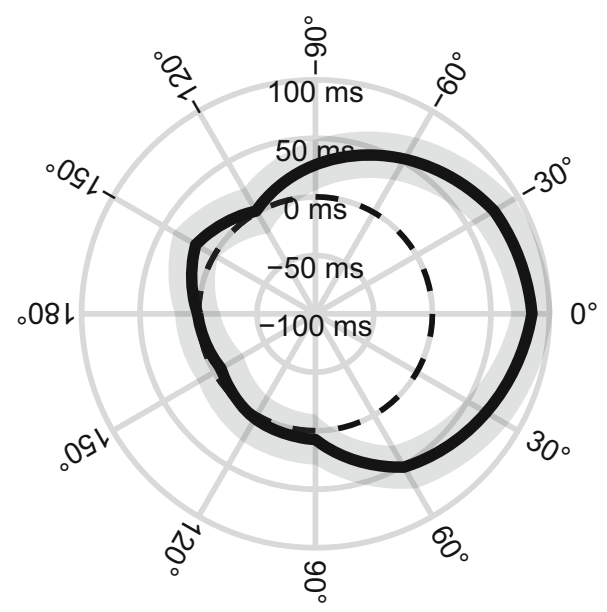

100 ms priming

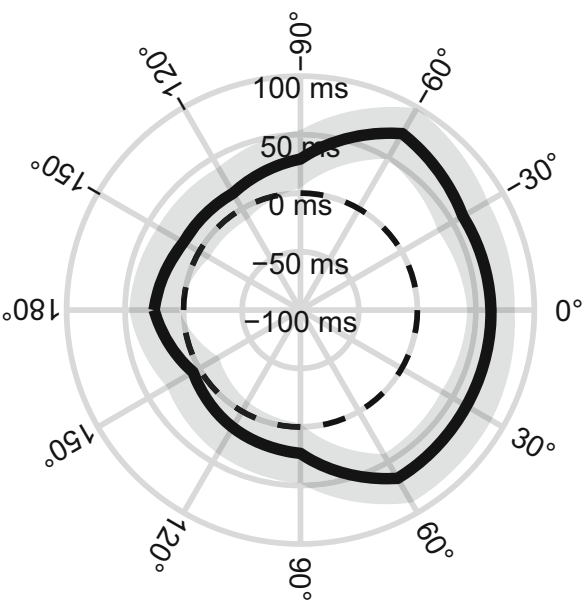

outside of this. Note that measurements were only made in the orientations denoted with ticks, and the lines between are interpolated for easier visual interpretation. Orientation labels also serve as exemplars for the indicated rotation

larger degree of rotation than expected by earlier evidence (Cohen et al., 2008; Dehaene et al., 2005; but challenged by Perea et al., 2018; Witzel et al., 2011). As for the $-150^{\circ}$ priming effect at 75-ms duration and the upside-down effect in 75 and $100-\mathrm{ms}$ durations, we cannot yet provide feasible explanations.

These discrepancies with the LCD model might point us to different models of word recognition. The SERIOL model for instance predicts that degradation, in our case rotation and probably the shortness of the presentation, affects the letterlevel representation and limits the number of characters that can be extracted during an oscillatory cycle (the temporal quantum of word processing, proposed at $40 \mathrm{~Hz}$; Whitney, 2002). The prime durations tested in our first experiment coincide with $1,2,3$, and 4 of such cycles, and it would be 


\section{Reversed prime}

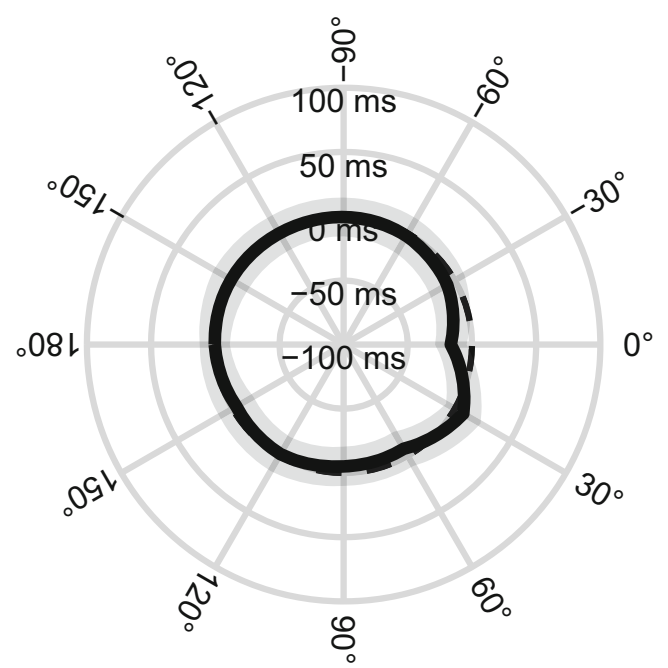

Fig. 3 Priming effect as a function of prime orientation in Experiment 2. RT difference between marginal means of "same" and "different" conditions in $\mathrm{ms} \pm 95 \%$ confidence intervals. The dashed circle is the zero line, meaning no effect, and positive priming effects are observed outside of this. Note that measurements were only made in the orientations denoted with ticks, and the lines between are interpolated for easier visual interpretation. Orientation labels also serve as exemplars for the indicated rotation

interesting to examine whether this model could account for the priming effects we measured.

Experiment 2 yielded two important supplementary findings. Firstly, the effects seen in the 50-ms group of Experiment 1 cannot be explained by the repeated exposure to the same letters alone, as there was no facilitative priming at all when the letter content was unchanged but the code was broken. Secondly, it was shown earlier that individual letter identification is unaffected by extreme rotations $\left(\geq 90^{\circ}\right.$; Perea et al., 2020); if we assume that a string of rotated letters can be processed similarly by the decoding system, we would expect to see some priming effect with upside-down presented reversed primes (reversal with $180^{\circ}$ rotation fixes the relative letter order). As this was not the case, we propose that internal cues of letter orientation could also serve as cues of word orientation, a potentially important piece of the code. To find the letter order, one must also find the start of the word and extract ordinal information along the axis defined by it. The default axis depends on the writing system, but also on the reference frame that the observer perceives, which depends on multiple factors (body position, visual background, and direction of gravity, according to Dyde, Jenkin, \& Harris, 2006). The prevalence of accented characters in the Hungarian alphabet could be an important source of information on word orientation, as accents are only present on top of letters (approximately one-third of the words used as stimuli in this study contain such characters).
Regarding the inverse priming effect observed with reversed primes in the normal orientation, we think it might be explained by the inhibitory effect of "reversed anagram" priming, observed by Morris and Still (2012). In their case, a similar prime can elicit an inhibitory effect, when despite the similarity, there is strong evidence for a different word identity. Although our reversal retains the letter content, and maybe even the inner part of the code (Davis \& Lupker, 2017), the mismatch at the more weighted starting and final letter positions could create competition instead of facilitation.

Finally, from a network point of view, we hypothesize that at all times, multiple systems attempt to make sense of the same sensory inputs, but only one perception can enter the consciousness: the one predicted with the highest confidence. When all systems are pointing at the same result, they increase fidelity. However, if they are conflicting, the perception could be unstable, and if certainty is low, more time is needed for slower systems to reach a decision boundary. This could be the reason why we see a continuous slowing with rotated words when the targets are manipulated (Cohen et al., 2008), but a stable effect in our priming paradigm. In this case, no matter how unsure the VWFA is in its prediction about the identity of the prime, if it was made, a priming effect follows. We propose that our results reflect the limitations of the VWFA as a separate system more precisely, whereas the results of Cohen et al. (2008) are more descriptive of the whole reading network.

\section{Conclusions}

We found that it is possible to obtain a significant priming effect with rotated prime words in a reading task. The most important explanatory factor of RT was found to be the interaction of prime identity and orientation. This is in contrast with the rotated priming effect in object naming, which was found to be invariant over orientation (Harris et al., 2008).

The extent of rotation that the effect resists is somewhat dependent upon priming duration. However, with priming durations above $50 \mathrm{~ms}$, priming has a significant effect up to $\pm 60^{\circ}$ of in-plane rotation and in some cases even above. This suggests that the automated mechanisms responsible for the decoding of visual words could be more robust than previously suggested (Cohen et al., 2008).

When using a reversed letter order in the primes, the effects vanished, so the same letter content is not sufficient to elicit effective priming in any orientation. We conclude that the effects seen with normal prime words come from higher orthographic processing levels, even when the primes are rotated.

There is a certain limitation to how isolated word recognition experiments can be translated to the more complex activity of natural reading. In recent years, there were some 


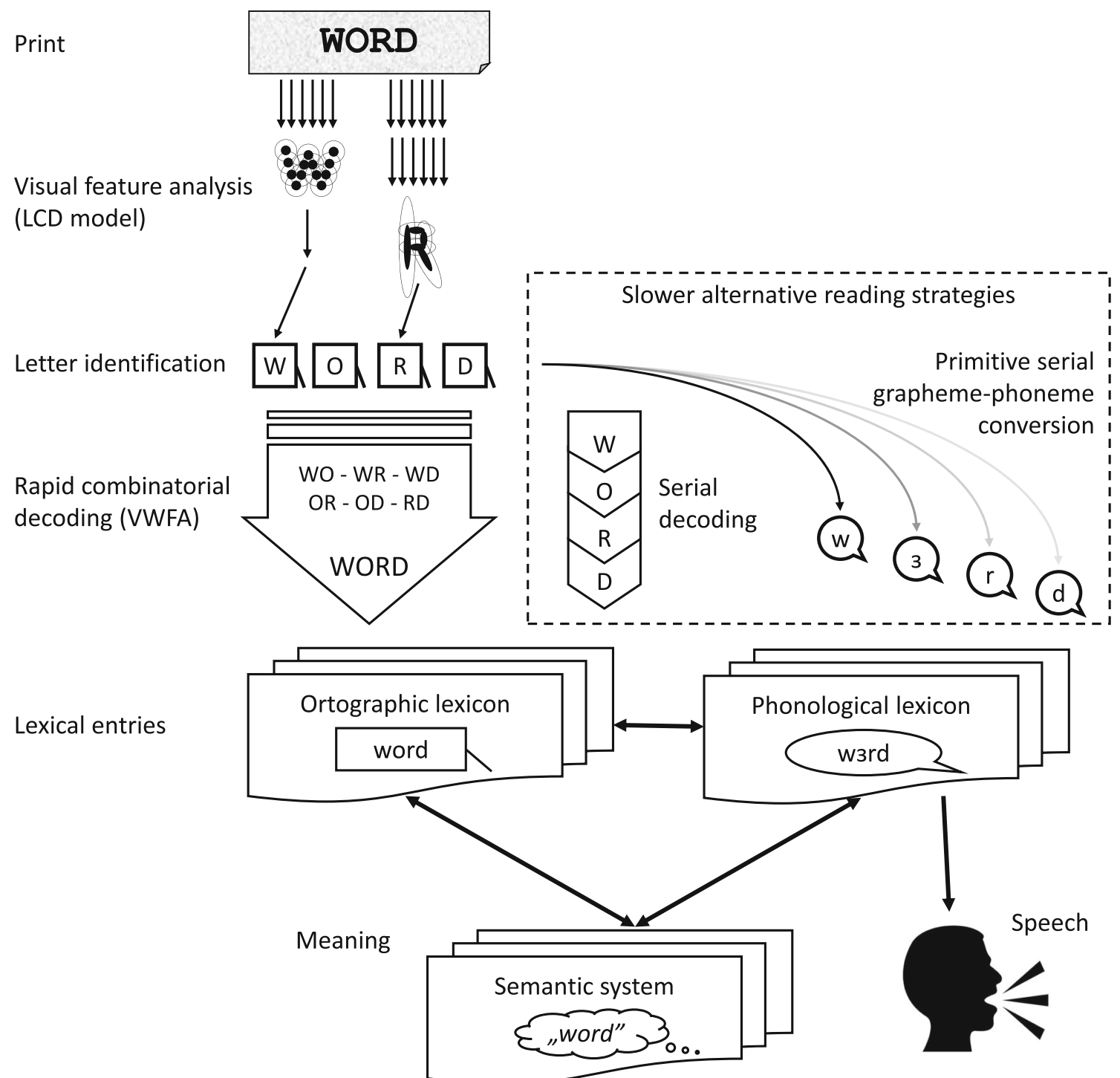

Fig. 4 Schematic model of word form and lexical processing in our experiments, based on the LCD model (Dehaene et al., 2005) and the figures of Ramus (2004) on reading acquisition. The VWFA, trained for orthographic regularities, extracts the code in an automated processing step. When this is incapacitated, decoding is aided by slower, more attentive processes exemplary studies using rotated letter words in both primed and unprimed settings of natural reading (Blythe, Juhasz, Tbaily, Rayner, \& Liversedge, 2019; Fernández-Lopez, Mirault, Grainger, \& Perea, in press). In our future work, we hope to follow them in extending our findings to a more native context.

\section{References}

Bates, D., Mächler, M., Bolker, B., \& Walker, S. (2015). Fitting linear mixed-effects models using lme4. Journal of Statistical Software, 67(1), 1-48. https://doi.org/10.18637/jss.v067.i01

Blythe, H. I., Juhasz, B. J., Tbaily, L. W., Rayner, K., \& Liversedge, S. P. (2019). Reading sentences of words with rotated letters: An eye movement study. Quarterly Journal of Experimental Psychology
(2006), 72(7), 1790-1804. https://doi.org/10.1177/ 1747021818810381

Bouhali, F., Thiebaut de Schotten, M., Pinel, P., Poupon, C., Mangin, J.F., Dehaene, S., \& Cohen, L. (2014). Anatomical connections of the visual word form area. The Journal of Neuroscience, 34(46), 15402-15414. https://doi.org/10.1523/JNEUROSCI.4918-13.2014

Cohen, L., Dehaene, S., Naccache, L., Lehéricy, S., Dehaene-Lambertz, G., Hénaff, M.-A., \& Michel, F. (2000). The visual word form area: Spatial and temporal characterization of an initial stage of reading in normal subjects and posterior split-brain patients. Brain, 123(2), 291-307. https://doi.org/10.1093/brain/123.2.291

Cohen, L., Dehaene, S., Vinckier, F., Jobert, A., \& Montavont, A. (2008). Reading normal and degraded words: Contribution of the dorsal and ventral visual pathways. NeuroImage, 40(1), 353-366. https://doi. org/10.1016/j.neuroimage.2007.11.036

Corballis, M. C., \& Armstrong, C. (2007). Repetition blindness is orientation blind. Memory \& Cognition, 35(2), 372-380. https://doi.org/ 10.3758/BF03193458 
Davis, C. J., \& Lupker, S. J. (2017). A backwards glance at words: Using reversed-interior masked primes to test models of visual word identification. PLOS ONE, 12(12), Article e0189056. https://doi.org/10. 1371/journal.pone.0189056

Dehaene, S., \& Cohen, L. (2007). Cultural Recycling of Cortical Maps. Neuron, 56(2), 384-398. https://doi.org/10.1016/j.neuron.2007.10. 004

Dehaene, S., \& Cohen, L. (2011). The unique role of the visual word form area in reading. Trends in Cognitive Sciences, 15(6), 254-262. https://doi.org/10.1016/j.tics.2011.04.003

Dehaene, S., Cohen, L., Sigman, M., \& Vinckier, F. (2005). The neural code for written words: A proposal. Trends in Cognitive Sciences, 9(7), 335-341. https://doi.org/10.1016/j.tics.2005.05.004

Dehaene, S., Naccache, L., Cohen, L., Bihan, D. L., Mangin, J.-F., Poline, J.-B., \& Rivière, D. (2001). Cerebral mechanisms of word masking and unconscious repetition priming. Nature Neuroscience, 4(7), 752-758. https://doi.org/10.1038/89551

Dejerine, J. (1892). Contribution à l'étude anatomopathologique et clinique des différents variétés de cécité verbal [Contribution to the anatomopathological and clinical study of the different varieties of verbal blindness]. Mémoires de La Société de Biologie, 4, 61-90.

Dyde, R. T., Jenkin, M. R., \& Harris, L. R. (2006). The subjective visual vertical and the perceptual upright. Experimental Brain Research, 173(4), 612-622. https://doi.org/10.1007/s00221-006-0405-y

Fernández-Lopez, M., Mirault, J., Grainger, J., \& Perea, M. (in press). How resilient is reading to letter rotations? A parafoveal preview investigation. Journal of Experimental Psychology: Learning, Memory, \& Cognition.

Grainger, J. (2018). Orthographic processing: A 'mid-level' vision of reading: The 44th Sir Frederic Bartlett Lecture. Quarterly Journal of Experimental Psychology, 71(2), 335-359. https://doi.org/10. 1080/17470218.2017.1314515

Harris, I. M., Dux, P. E., Benito, C. T., \& Leek, E. C. (2008). Orientation sensitivity at different stages of object processing: Evidence from repetition priming and naming. PLOS ONE, 3(5), e2256. https://doi. org/10.1371/journal.pone.0002256

Harris, I. M., Harris, J. A., \& Caine, D. (2001). Object orientation agnosia: A failure to find the axis? Journal of Cognitive Neuroscience, 13(6), 800-812. https://doi.org/10.1162/08989290152541467

Holcomb, P. J., \& Grainger, J. (2007). Exploring the temporal dynamics of visual word recognition in the masked repetition priming paradigm using event-related potentials. Brain Research, 1180, 39-58. https://doi.org/10.1016/j.brainres.2007.06.110

Kim, J. S., Kanjlia, S., Merabet, L. B., \& Bedny, M. (2017). Development of the visual word form area requires visual experience: Evidence from blind braille readers. Journal of Neuroscience, 37(47), 1149511504. https://doi.org/10.1523/JNEUROSCI.0997-17.2017

Kleiner, M., Brainard, D., \& Pelli, D. (2007). What's new in Psychtoolbox-3? Perception, 36(14), 1-16.

Koriat, A., \& Norman, J. (1989). Why is word recognition impaired by disorientation while the identification of single letters is not? Journal of Experimental Psychology: Human Perception and
Performance, 15(1), 153-163. https://doi.org/10.1037//0096-1523. 15.1.153

Lenth, R., Singmann, H., Love, J., Buerkner, P., \& Herve, M. (2018). Emmeans: Estimated marginal means, aka least-squares means ( $\mathrm{R}$ Package Version, 1.1.3). https://CRAN.R-project.org/package $=$ emmeans

Martin, A. (2006). Shades of Déjerine-Forging a causal link between the visual word form area and reading. Neuron, 50(2), 173-175. https://doi.org/10.1016/j.neuron.2006.04.004

Morris, A. L., \& Still, M. L. (2012). Orthographic similarity: The case of "reversed anagrams." Memory \& Cognition, 40(5), 779-790. https:// doi.org/10.3758/s13421-012-0183-7

Norris, D. (2013). Models of visual word recognition. Trends in Cognitive Sciences, 17(10), 517-524. https://doi.org/10.1016/j.tics. 2013.08.003

Oravecz, C., Váradi, T., \& Sass, B. (2014). The Hungarian gigaword corpus. Proceedings of the Ninth International Conference on Language Resources and Evaluation (LREC'14; pp. 1719-1723). http://www.lrec-conf.org/proceedings//rec2014/pdf/681_Paper.pdf

Perea, M., Marcet, A., \& Fernández-López, M. (2018). Does letter rotation slow down orthographic processing in word recognition? Psychonomic Bulletin \& Review, 25(6), 2295-2300.

Perea, M., Vergara-Martínez, M., Marcet, A., Mallouh, R. A., \& Fernández-López, M. (2020). When does rotation disrupt letter encoding? Testing the resilience of letter detectors in the initial moments of processing. Memory \& Cognition, 1-6. Advance online publication. https://doi.org/10.3758/s13421-020-01013-9

Ramus, F. (2004). The neural basis of reading acquisition. In M. S. Gazzaniga (Ed.), The cognitive neurosciences (3rd ed., pp. 815824). MIT Press. http://www.lscp.net/persons/ramus/docs/TCNS3. pdf

Reich, L., Szwed, M., Cohen, L., \& Amedi, A. (2011). A ventral visual stream reading center independent of visual experience. Current Biology, 21(5), 363-368. https://doi.org/10.1016/j.cub.2011.01.040

Risko, E. F., Medimorec, S., Chisholm, J., \& Kingstone, A. (2014). Rotating with rotated text: A natural behavior approach to investigating cognitive offloading. Cognitive Science, 38(3), 537-564. https://doi.org/10.1111/cogs.12087

Warrington, E. K., \& Shallice, T. I. M. (1980). Word-form dyslexia. Brain: A Journal of Neurology, 103(1), 99-112.

Whitney, C. (2002). An explanation of the length effect for rotated words. Cognitive Systems Research, 3(1), 113-119. https://doi.org/10. 1016/S1389-0417(01)00050-X

Wickham, H. (2016). ggplot2: Elegant graphics for data analysis. Springer.

Witzel, N., Qiao, X., \& Forster, K. (2011). Transposed letter priming with horizontal and vertical text in Japanese and English readers. Journal of Experimental Psychology: Human Perception and Performance, 37(3), 914-920. https://doi.org/10.1037/a0022194

Publisher's note Springer Nature remains neutral with regard to jurisdictional claims in published maps and institutional affiliations. 